\title{
Bipartite entanglement in fermion systems
}

\author{
N. Gigena, R. Rossignoli \\ IFLP-Departamento de Física-FCE, Universidad Nacional de La Plata, C.C. 67, La Plata (1900), Argentina
}

\begin{abstract}
We discuss the relation between fermion entanglement and bipartite entanglement. We first show that an exact correspondence between them arises when the states are constrained to have a definite local number parity. Moreover, for arbitrary states in a four dimensional single-particle Hilbert space, the fermion entanglement is shown to measure the entanglement between two distinguishable qubits defined by a suitable partition of this space. Such entanglement can be used as a resource for tasks like quantum teleportation. On the other hand, this fermionic entanglement provides a lower bound to the entanglement of an arbitrary bipartition although in this case the local states involved will generally have different number parities. Finally the fermionic implementation of the teleportation and superdense coding protocols based on qubits with odd and even number parity is discussed, together with the role of the previous types of entanglement.

pacs03.67.Mn, 03.65.Ud, 05.30.Fk
\end{abstract}

\section{INTRODUCTION}

Entanglement is a fundamental feature of quantum mechanics, and its quantification and characterization has been one of the main goals of quantum information theory for the last decades [1]. It is also at the heart of quantum information processing [4], being recognized as the key ingredient for quantum state teleportation [5] and the resource that makes some pure state based quantum algorithms exponentially faster than their classical counterparts [6].

Although entanglement has been extensively studied for systems of distinguishable constituents, less attention has been paid to the case of a system of indistinguishable fermions. Only in recent years the topic has gained an increasing strength [26]. Mainly two different approaches may be recognized in the attempts of generalizing the definition of entanglement to fermion systems: The first is entanglement between modes [13 17, where the system and subsystems consist of some collection of single-particle modes that can be shared. This approach requires to fix some basis of the single-particle state space and then to specify the modes that constitute each subsystem. The other approach is known as entanglement between particles 7 12, 18 23, 25, where the indistinguishable constituents of the system are taken as subsystems and entanglement is defined beyond symmetrization.

In a previous work 24 we defined an entropic measure of mode entanglement in fermion systems which is shown to be a measure of entanglement between particles after an optimization over bases of the single-particle (sp) state space is performed. Moreover, when the sp state space dimension is four and the particle number is fixed to two, this entanglement measure reduces to the Slater correlation measure defined in 7]. In the present work we first show that the entanglement between two distinguishable qubits is the same as that measured by this fermionic entanglement entropy when the fermionic states are constrained to have a fixed local number parity in the associated bipartition of the sp space. Then we use this correspondence to show that, in fact, any state of a fermion system with a 4-dimensional sp Hilbert space may be seen as a state of two distinguishable qubits for a suitable bipartition of the sp space, with its entanglement measured by the fermionic entanglement entropy. On the other hand, for an arbitrary bipartition involving no fixed local number parity the fermionic entanglement entropy is shown to provide a lower bound to the associated bipartite entanglement. As application we use these results to show that qubit-based quantum circuits may be rewritten as mode-based fermionic circuits if we impose the appropriate restriction to the occupation numbers, recovering reversible classical computation when the input states are Slater determinants (in the basis of interest). Two types of fermionic qubit representations, based on odd or even number parity qubits, are seen to naturally emerge. Finally, we show that the extra bipartite entanglement that can be obtained by relaxing this local parity restriction can in principle be used for protocols such as superdense coding.

The formalism and theoretical results are provided in sec. II while their applications are discussed in sec. III Conclusions are finally provided in sec. IV

\section{FORMALISM}

\section{A. Fermionic entanglement entropy and concurrence}

We will consider a fermion system with a single-particle (sp) Hilbert space $\mathcal{H}$. We will deal with pure states $|\psi\rangle$ which do not necessarily have a fixed particle number, although the number parity will be fixed, in agreement with the parity superselection rule [27]: $P|\psi\rangle= \pm|\psi\rangle$, with $P=\exp \left[i \pi \sum_{j} c_{j}^{\dagger} c_{j}\right]$ the number parity operator. Here $c_{j}, c_{j}^{\dagger}$ denote fermion annihilation and creation operators satisfying the usual anticommutation relations

$$
\left\{c_{i}, c_{j}\right\}=0, \quad\left\{c_{i}, c_{j}^{\dagger}\right\}=\delta_{i j} .
$$

In 24] we defined a one-body entanglement entropy for 
a general pure fermion state $|\psi\rangle$,

$$
S^{\mathrm{sp}}(|\psi\rangle)=\operatorname{Tr} h\left(\rho^{\mathrm{sp}}\right),
$$

where $\rho_{i j}^{\mathrm{sp}}=\left\langle c_{j}^{\dagger} c_{i}\right\rangle \equiv\left\langle\psi\left|c_{j}^{\dagger} c_{i}\right| \psi\right\rangle$ is the one body density matrix of the system and $h(p)=-p \log _{2} p-(1-$ p) $\log _{2}(1-p)$. Eq. (2) is proportional to the minimum, over all sp bases of $\mathcal{H}$, of the average entanglement entropy between a sp mode and its orthogonal complement (which in turn arises from a properly defined measurement of the occupation of a sp mode), and vanishes iff $|\psi\rangle$ is a Slater Determinant (SD), i.e. $|\psi\rangle=c_{1}^{\dagger} \ldots c_{k}^{\dagger}|0\rangle$. This definition is easily extended to quasiparticle modes, in which case 24]

$$
S^{\mathrm{qsp}}(|\psi\rangle)=-\operatorname{Tr} \rho^{\mathrm{qsp}} \log _{2}\left(\rho^{\mathrm{qsp}}\right),
$$

where $\rho^{\text {qsp }}$ is now the extended one-body density matrix

$$
\rho^{\mathrm{qsp}}=1-\left\langle\left(\begin{array}{c}
\boldsymbol{c} \\
\boldsymbol{c}^{\dagger}
\end{array}\right)\left(\begin{array}{ll}
\boldsymbol{c}^{\dagger} & \boldsymbol{c}
\end{array}\right)\right\rangle=\left(\begin{array}{cc}
\rho^{\mathrm{sp}} & \kappa \\
-\bar{\kappa} & \mathbb{1}-\bar{\rho}^{\mathrm{sp}}
\end{array}\right),
$$

with $\kappa_{i j}=\left\langle c_{j} c_{i}\right\rangle,-\bar{\kappa}_{i j}=\left\langle c_{j}^{\dagger} c_{i}^{\dagger}\right\rangle$ and $\left(\mathbb{1}-\bar{\rho}^{\mathrm{sp}}\right)_{i j}=\left\langle c_{j} c_{i}^{\dagger}\right\rangle$. Eq. (3) vanishes iff $|\psi\rangle$ is a quasiparticle vacuum or SD and satisfies $S^{\mathrm{qsp}}(|\psi\rangle) \leq S^{\mathrm{sp}}(|\psi\rangle)$, with $S^{\mathrm{qsp}}(|\psi\rangle)=$ $S^{\mathrm{sp}}(|\psi\rangle)$ iff $\kappa=0$.

While Eq. (2) is invariant under unitary transformations $c_{i} \rightarrow \sum_{k} \bar{U}_{k i} c_{k}, U U^{\dagger}=I$, which lead to $\rho^{\mathrm{sp}} \rightarrow$ $U^{\dagger} \rho^{\mathrm{sp}} U$, Eq. (4) remains invariant under general Bogoliubov transformations

$$
c_{i} \rightarrow a_{i}=\sum_{k} \bar{U}_{k i} c_{k}+V_{k i} c_{k}^{\dagger},
$$

where matrices $U$ and $V$ satisfy $U U^{\dagger}+V V^{\dagger}=\mathbb{1}$ and $U V^{T}+V U^{T}=0$ in order that $\left\{a_{i}, a_{i}^{\dagger}\right\}$ fulfill the fermionic anticommutation relations 28]. In this case $\rho^{\mathrm{qsp}} \rightarrow$ $W^{\dagger} \rho^{\mathrm{qsp}} W$, with $W=\left(\frac{U}{\bar{V}} \bar{U}\right)$ a unitary matrix. In terms of the operators diagonalizing $\rho^{\mathrm{qsp}}$, we then have

$$
1-\left\langle\left(\begin{array}{c}
\boldsymbol{a} \\
\boldsymbol{a}^{\dagger}
\end{array}\right)\left(\begin{array}{ll}
\boldsymbol{a}^{\dagger} & \boldsymbol{a}
\end{array}\right)\right\rangle=\left(\begin{array}{cc}
f & 0 \\
0 & 1-f
\end{array}\right),
$$

with $f_{k l}=f_{k} \delta_{k l}$ and $f_{k}, 1-f_{k}$ the eigenvalues of $\rho^{\mathrm{qsp}}$.

For a sp space $\mathcal{H}$ of dimension $4, \rho^{\text {qsp }}$ becomes an $8 \times 8$ matrix, and it was shown that its eigenvalues for a pure state $|\psi\rangle$ are fourfold degenerate and can be written as 24.

$$
f_{ \pm}=\frac{1 \pm \sqrt{1-C^{2}(|\psi\rangle)}}{2},
$$

where $C(|\psi\rangle)=2 \sqrt{f_{+} f_{-}} \in[0,1]$ is called fermionic concurrence, in analogy with that defined for two-qubits [29]. Eq. (3) becomes then an increasing function of $C(|\psi\rangle)$, vanishing iff the latter vanishes. This fermionic concurrence can also be explicitly evaluated: Writing a general even number parity pure state in such a space as

$$
|\psi\rangle=\left(\alpha_{0}+\frac{1}{2} \sum_{i, j} \alpha_{i j} c_{i}^{\dagger} c_{j}^{\dagger}+\alpha_{4} c_{1}^{\dagger} c_{2}^{\dagger} c_{3}^{\dagger} c_{4}^{\dagger}\right)|0\rangle,
$$

where $\alpha_{i j}=-\alpha_{j i}, i, j=1, \ldots, 4$ and $\left|\alpha_{0}^{2}\right|+\left|\alpha_{4}^{2}\right|+$ $\frac{1}{2} \operatorname{tr} \alpha^{\dagger} \alpha=1$, then $\rho^{\mathrm{sp}}=\alpha \alpha^{\dagger}+\left|\alpha_{4}\right|^{2} \mathbb{1}, \kappa=\alpha_{0}^{*} \alpha+\alpha_{4} \tilde{\alpha}^{*}$, with $\tilde{\alpha}_{i j}=\frac{1}{2} \sum_{k, l} \epsilon_{i j k l} \alpha_{k l}\left(\epsilon_{i j k l}\right.$ denotes the fully antisymmetric tensor) and it can be shown that 24.

$$
C(|\psi\rangle)=2\left|\alpha_{12} \alpha_{34}-\alpha_{13} \alpha_{24}+\alpha_{14} \alpha_{23}-\alpha_{0} \alpha_{4}\right| .
$$

For two-fermion states $\left(\alpha_{0}=\alpha_{4}=0\right)$ it reduces to the Slater correlation measure defined in [, 9, for which $\kappa=$ 0 and $f_{ \pm}$become the eigenvalues (two-fold degenerate) of $\rho^{\mathrm{sp}}$. An expression similar to (8) holds for an odd number parity state (see 24] and sec. [IE). Moreover, in such sp space the concurrence and the associated entanglement of formation can also be explicitly determined for arbitrary mixed states 7,24 .

A four-dimensional sp space (which generates an eightdimensional state space for each value of the parity $P$ ) becomes then exactly solvable, being as well the first nontrivial dimension since for $\operatorname{dim} \mathcal{H} \leq 3$ any definite parity pure state can be written as a SD or quasiparticle vacuum 24], as verified from (8) $(C(|\psi\rangle)=0$ if one of the sp sates is left empty). It is also physically relevant, since it can accommodate the basic situation of two spin $1 / 2$ fermions at two different sites or, more generally, states of spin $1 / 2$ fermions occupying just two orbital states, as in a double well scenario. The relevant sp space in these cases is $\mathcal{H}_{\mathcal{S}} \otimes \mathcal{H}_{\mathcal{O}}$, with $\mathcal{H}_{\mathcal{S}}$ the spin space and $\mathcal{H}_{\mathcal{O}}$ the twodimensional subspace spanned by the two orbital states. In particular, just four sp states are essentially used in recent proposals for observing Bell-violation from single electron entanglement 30].

\section{B. Bipartite entanglement as two-fermion entanglement}

Let us now consider a system of two distinguishable qubits prepared in a pure state $\alpha_{+}|00\rangle+\alpha_{-}|11\rangle$, i.e.

$$
|\psi\rangle_{A B}=\alpha_{+}|\uparrow\rangle_{A} \otimes|\uparrow\rangle_{B}+\alpha_{-}|\downarrow\rangle_{A} \otimes|\downarrow\rangle_{B},
$$

where $\left|\alpha_{+}^{2}\right|+\left|\alpha_{-}^{2}\right|=1$ and the notation indicates a possible realization in terms of two spin $1 / 2$ particles located at different sites $A, B$, with their spins aligned parallel or antiparallel to a given $(z)$ axis. We can also consider this last state as a two-fermion state of a spin $1 / 2$ fermion system with sp space $\mathcal{H}=\mathcal{H}_{\mathcal{S}} \otimes \mathcal{H}_{\mathcal{O}}$ :

$$
|\psi\rangle_{f}=\left(\alpha_{+} c_{A \uparrow}^{\dagger} c_{B \uparrow}^{\dagger}+\alpha_{-} c_{A \downarrow}^{\dagger} c_{B \downarrow}^{\dagger}\right)|0\rangle,
$$

with $|0\rangle$ the fermionic vacuum. A measurement of spin " $A$ " or " $B$ " along $z$ can be described in the fermionic representation by the operators $\Pi_{S \mu}=$ $c_{S \mu}^{\dagger} c_{S \mu}, S=A, B, \mu=\uparrow, \downarrow$, which satisfy $\Pi_{S \mu}^{2}=\Pi_{S \mu}$ and $\left[\Pi_{S \mu}, \Pi_{S^{\prime} \mu^{\prime}}\right]=0$, with $\sum_{\mu} \Pi_{S \mu}|\psi\rangle_{f}=|\psi\rangle_{f}$. Furthermore, we can describe any "local" operator on $A$ or 
$B$ in terms of Pauli operators if we define, for $S=A, B$,

$$
\begin{aligned}
\sigma_{S x} & =c_{S \uparrow}^{\dagger} c_{S \downarrow}+c_{S \downarrow}^{\dagger} c_{S \uparrow}, \\
\sigma_{S y} & =-i\left(c_{S \uparrow}^{\dagger} c_{S \downarrow}-c_{S \downarrow}^{\dagger} c_{S \uparrow}\right), \\
\sigma_{S z} & =c_{S \uparrow}^{\dagger} c_{S \uparrow}-c_{S \downarrow}^{\dagger} c_{S \downarrow},
\end{aligned}
$$

which verify the usual commutation relations $\left[\sigma_{S j}, \sigma_{S^{\prime} k}\right]=2 i \delta_{S S^{\prime}} \epsilon_{j k l}, \quad\left(\epsilon_{j k l}\right.$ is the antisymmetric tensor), with $\sigma_{S j}^{2}|\psi\rangle_{f}=|\psi\rangle_{f}$.

It is also apparent that the state (9) is separable iff $\alpha_{+}=0$ or $\alpha_{-}=0$, which is precisely the condition which ensures that the state (10) is a SD. Moreover, the standard concurrence 29] of the state (9) is identical with the fermionic concurrence (8) of the state (10):

$$
C\left(|\psi\rangle_{A B}\right)=2\left|\alpha_{+} \alpha_{-}\right|=C\left(|\psi\rangle_{f}\right),
$$

with $f_{ \pm}=\left|\alpha_{ \pm}^{2}\right|$ in (6). Entangled two-qubit states (9) correspond then to two-fermion states (10) which are not SD's, and vice-versa.

Such correspondence remains of course valid for any bipartite two-qubit state

$$
|\psi\rangle_{A B}=\sum_{\mu, \nu} \alpha_{\mu \nu}|\mu\rangle_{A} \otimes|\nu\rangle_{B}
$$

which in the fermionic representation becomes

$$
|\psi\rangle_{f}=\sum_{\mu, \nu} \alpha_{\mu \nu} c_{A \mu}^{\dagger} c_{B \nu}^{\dagger}|0\rangle
$$

We now obtain $C\left(|\psi\rangle_{A B}\right)=2|\operatorname{det} \alpha|=C\left(|\psi\rangle_{f}\right)$, according to the standard and fermionic (Eq. (8) ) expressions. These states can in fact be taken to the previous Schmidt forms (9)-10) (with $\left|\alpha_{ \pm}\right|$the singular values of the matrix $\alpha$ ) by means of local unitary transformations, which in the fermionic representation become $c_{S \mu} \rightarrow \sum_{\nu} \bar{U}_{\nu \mu}^{S} c_{S \nu}$.

Previous considerations remain also valid for general bipartite states of systems of arbitrary dimension $(\mu=$ $1, \ldots, d_{A}, \nu=1, \ldots, d_{B}$ in (13)-(14)), if the sp space of the associated fermionic system (of dimension $d_{A}+d_{B}$ ) is decomposed as $\mathcal{H}_{A} \oplus \mathcal{H}_{B}$. The sp density matrix $\rho^{\text {sp }}$ derived from the state (14) takes in the general case the blocked form

$$
\rho^{\mathrm{sp}}=\left(\begin{array}{cc}
\alpha \alpha^{\dagger} & 0 \\
0 & \alpha^{T} \bar{\alpha}
\end{array}\right)=\left(\begin{array}{cc}
\rho_{A} & 0 \\
0 & \rho_{B}
\end{array}\right),
$$

i.e. $\left\langle c_{S \nu}^{\dagger} c_{S^{\prime} \mu}\right\rangle=\delta_{S S^{\prime}}\left(\rho_{S}\right)_{\mu \nu}$, where $\rho_{A(B)}$ are the local reduced density matrices $\operatorname{Tr}_{B(A)}|\psi\rangle_{A B}\langle\psi|$ of the state (13) in the standard basis. Hence, in the fermionic setting $\rho^{\mathrm{sp}}$ contains the information of both local states and its diagonalization implies that of both $\rho_{A}$ and $\rho_{B}$. Its eigenvalues will then be those of these matrices, being hence two-fold degenerate and equal to the square of the singular values of the matrix $\alpha$ (becoming $f_{ \pm}=\left|\alpha_{ \pm}\right|^{2}$ in the two-qubit case). In the general case, the entanglement entropy of the sate (13) can then be written as

$$
E(A, B)=S\left(\rho_{A}\right)=S\left(\rho_{B}\right)=\frac{1}{2} S\left(\rho^{\mathrm{sp}}\right),
$$

which holds for the von Neumann entropy $S(\rho)=$ $-\operatorname{Tr} \rho \log _{2} \rho$ as well as for any trace form entropy 31] $S(\rho)=\operatorname{Tr} f(\rho)(f$ concave, $f(0)=f(1)=0)$. Thus, the entanglement entropy of the general bipartite state (13) is just proportional to the fermionic entanglement entropy (as defined in (2)) of the associated state (14). Hence, for any dimension there is an exact correspondence between the bipartite states (13) and the two-fermion states (14), with local operators represented by linear combinations of one-body local fermion operators $c_{S \nu}^{\dagger} c_{S \mu}$ (satisfying $\left.\left[c_{A \mu}^{\dagger} c_{A \nu}, c_{B \mu^{\prime}}^{\dagger} c_{B \nu^{\prime}}\right]=0\right)$ and $|\psi\rangle_{A B}$ entangled iff $|\psi\rangle_{f}$ is not a SD.

This equivalence holds also for mixed states i.e., convex combinations of the states (13) and (14). The bipartite states will be separable, i.e., convex combinations of product states 32] iff the associated fermionic mixed state can be written as a convex combination of SD's of the form (14). In particular, for two-qubit states a fourdimensional sp fermion space suffices and the standard mixed state concurrence 29] will coincide exactly with the fermionic mixed state concurrence [7, 20 22, 24] of mixtures of states (14).

\section{Bipartite entanglement as quasiparticle fermion entanglement}

Other fermionic representations of the state (13) with similar properties are also feasible. For instance, in the two qubit case we can perform a particle holetransformation of the fermion operators with spin down,

$$
c_{S \uparrow}^{\dagger} \longrightarrow c_{S \uparrow}^{\dagger}, \quad c_{S \downarrow}^{\dagger} \longrightarrow c_{S \downarrow}, \quad S=A, B
$$

such that the aligned state $|\downarrow\rangle_{A} \otimes|\downarrow\rangle_{B}$ corresponds now to the vacuum of the new operators $\left(|0\rangle \longrightarrow c_{A \downarrow}^{\dagger} c_{B \downarrow}^{\dagger}|0\rangle\right)$, with the new $c_{S \downarrow}^{\dagger}$ creating a hole. The remaining states of the standard basis become one and two particle-hole excitations. We can then rewrite the state (10) as

$$
|\tilde{\psi}\rangle_{f}=\left(\alpha_{-}+\alpha_{+} c_{A \uparrow}^{\dagger} c_{A \downarrow}^{\dagger} c_{B \uparrow}^{\dagger} c_{B \downarrow}^{\dagger}\right)|0\rangle,
$$

i.e., as a superposition of the vacuum plus two particlehole excitations, with each "side" having now either 0 or two fermions, i.e., an even local number parity $\left(e^{i \pi N_{S}}=1\right.$ for $\left.S=A, B, N_{S}=\sum_{\mu} c_{S \mu}^{\dagger} c_{S \mu}\right)$. It is apparent that the state (18) is a a quasiparticle vacuum or SD iff $\alpha_{+}=0$ or $\alpha_{-}=0$. Moreover, for the state (18) Eq. (8) leads again to $C\left(|\tilde{\psi}\rangle_{f}\right)=2\left|\alpha_{+} \alpha_{-}\right|$, implying the equivalence (12) between the bipartite and the present generalized fermionic concurrrence, invariant under Bogoliubov (and hence particle-hole) transformations.

The local Pauli operators (11) become now

$$
\begin{aligned}
& \tilde{\sigma}_{S x}=c_{S \uparrow}^{\dagger} c_{S \downarrow}^{\dagger}+c_{S \downarrow} c_{S \uparrow}, \\
& \tilde{\sigma}_{S y}=-i\left(c_{S \uparrow}^{\dagger} c_{S \downarrow}^{\dagger}-c_{S \downarrow} c_{S \uparrow}\right), \\
& \tilde{\sigma}_{S z}=c_{S \uparrow}^{\dagger} c_{S \uparrow}+c_{S \downarrow}^{\dagger} c_{S \downarrow}-1,
\end{aligned}
$$


which verify the same $S U(2)$ commutation relations $\left[\tilde{\sigma}_{S j}, \tilde{\sigma}_{S^{\prime} k}\right]=2 i \delta_{S S^{\prime}} \epsilon_{j k l} \tilde{\sigma}_{S l}$, with $\tilde{\sigma}_{S j}^{2}|\tilde{\psi}\rangle_{f}=|\tilde{\psi}\rangle_{f} \forall j$. Any local operation can be written in terms of these operators, which represent now local paticle-hole creation or annihilation and counting.

Similarly, we may write the general two-qubit state (13) as

$$
|\tilde{\psi}\rangle_{f}=\sum_{\mu, \nu} \alpha_{\mu \nu}\left(c_{A \uparrow}^{\dagger} c_{A \downarrow}^{\dagger}\right)^{n_{\mu}}\left(c_{B \uparrow}^{\dagger} c_{B \downarrow}^{\dagger}\right)^{n_{\nu}}|0\rangle
$$

where $\mu, \nu= \pm$ and $n_{-}=0, n_{+}=1$. This state can be brought back to the "Schmidt" form (18) by means of "local" Bogoliubov transformations $c_{S \uparrow} \rightarrow u_{S} c_{S \uparrow}+$ $v_{S} c_{S \downarrow}^{\dagger}, c_{S \downarrow} \rightarrow u_{S} c_{S \downarrow}-v_{S} c_{S \uparrow}^{\dagger},\left|u_{S}^{2}\right|+\left|v_{S}^{2}\right|=1$, which will diagonalize $\rho^{\mathrm{qsp}}$ (see below) and change the vacuum as $|0\rangle \rightarrow\left[\prod_{S=A, B}\left(u_{S}-v_{S} c_{S \uparrow}^{\dagger} c_{S \downarrow}^{\dagger}\right)\right]|0\rangle$. It is again verified that for this state Eq. (8) leads to $C\left(|\tilde{\psi}\rangle_{f}\right)=2|\operatorname{det} \alpha|=$ $2\left|\alpha_{+} \alpha_{-}\right|$, with $\left|\alpha_{ \pm}\right|$the singular values of the matrix $\alpha$. The state (13) is then entangled iff the state (20) is not a quasiparticle vacuum or $\mathrm{SD}\left(C\left(|\tilde{\psi}\rangle_{f}\right)>0\right)$.

In this case the extended density matrix $\rho^{\mathrm{qsp}}$ is to be considered, with elements $\left\langle c_{S \nu}^{\dagger} c_{S^{\prime} \mu}\right\rangle=\delta_{S S^{\prime}} \delta_{\mu \nu} p_{S}$, $\left\langle c_{S \nu} c_{S^{\prime} \mu}\right\rangle=\delta_{S S^{\prime}} \delta_{\nu,-\mu}(-1)^{n_{\mu}} q_{S}$, where $p_{A(B)}=\left|\alpha_{++}\right|^{2}+$ $\left|\alpha_{+-(-+)}\right|^{2}, q_{A(B)}=\alpha_{++} \alpha_{-+(+-)}^{*}+\alpha_{+-(-+)} \alpha_{--}^{*}$. For the Schmidt form (20), $\rho^{\mathrm{qsp}}$ becomes diagonal $\left(\left(p_{A(B)}=\right.\right.$ $\left.\left|\alpha_{+}\right|^{2}, q_{A(B)}=0\right)$. Reduced states $\rho_{A(B)}$ are now to be recovered as particular blocks of $\rho^{\mathrm{qsp}}$ :

$$
\begin{aligned}
\rho_{S} & =\frac{1}{2}\left(\begin{array}{cc}
1+\left\langle\tilde{\sigma}_{S z}\right\rangle & \left\langle\tilde{\sigma}_{S x}\right\rangle-i\left\langle\tilde{\sigma}_{S y}\right\rangle \\
\left\langle\tilde{\sigma}_{S x}\right\rangle+i\left\langle\tilde{\sigma}_{S y}\right\rangle & 1-\left\langle\tilde{\sigma}_{S z}\right\rangle
\end{array}\right) \\
& =\left(\begin{array}{cc}
\left\langle c_{S \uparrow}^{\dagger} c_{S \uparrow}\right\rangle & \left\langle c_{S \downarrow} c_{S \uparrow}\right\rangle \\
\left\langle c_{S i \uparrow}^{\dagger} c_{S \downarrow}^{\dagger}\right\rangle & \left\langle c_{S \uparrow} c_{S \uparrow}^{\dagger}\right\rangle
\end{array}\right) .
\end{aligned}
$$

Diagonalization of $\rho^{\mathrm{qsp}}$ will, nevertheless, still imply that of $\rho_{A}$ and $\rho_{B}$. It is verified that its eigenvalues are $f_{ \pm}=$ $\left|\alpha_{ \pm}\right|^{2}$, four-fold degenerate, with $\left|\alpha_{ \pm}\right|$the singular values of the matrix $\alpha$. We then have

$$
E(A, B)=S\left(\rho_{A}\right)=S\left(\rho_{B}\right)=\frac{1}{4} S\left(\rho^{\mathrm{qsp}}\right),
$$

again valid for any trace-form entropy $S(\rho)=\operatorname{Tr} f(\rho)$. And for convex mixtures of states of the form (20) (whose rank will be at most 4), the mixed state fermionic concurrence, as defined in 24, will again coincide exactly with the standard two-qubit concurrence.

The same considerations hold for general bipartite states (13) of systems of arbitrary dimension if a particle hole transformation (or in general, a Bogoliubov transformation) is applied to the original fermion operators in (14). In such a case Eq. (22) is valid for entropic functions satisfying $f(p)=f(1-p)$ (a reasonable assumption as $p$ represents an average occupation number of particle or hole), since $\rho^{\text {qsp }}$ will have eigenvalues $f_{k}$ and $1-f_{k}$, now two-fold degenerate, with $f_{k}$ those of the local states $\rho_{A(B)}$.

A final remark is that the representations (11) and (19) of Pauli operators can coexist independently since

$$
\left[\sigma_{S j}, \tilde{\sigma}_{S^{\prime} k}\right]=0,
$$

$\forall j, k$ for both $S^{\prime} \neq S$ and $S^{\prime}=S(S U(2) \times S U(2)$ structure 33] at each side $A$ or $B$ ). Moreover, the even local parity states (20) belong to the kernel of the operators (11), while the odd local parity states (14) $\left(e^{i \pi N_{S}}=-1\right)$ belong to the kernel of the operators (19):

$$
\sigma_{S j}|\tilde{\psi}\rangle_{f}=\tilde{\sigma}_{S j}|\psi\rangle_{f}=0,
$$

for $S=A, B$ and $j=x, y, z$. Hence, unitary operators $e^{i \sum_{j} \lambda_{j} \sigma_{S j}}\left(e^{i \sum_{j} \lambda_{j} \tilde{\sigma}_{S j}}\right)$ will become identities when applied to states $|\tilde{\psi}\rangle_{f}\left(|\psi\rangle_{f}\right)$. A fermion system with a sp space of dimension 4 can then accommodate two distinct two-qubit systems, one for each value of the local number parity, keeping the total number parity fixed $\left(e^{i \pi\left(N_{A}+N_{B}\right)}=1\right)$.

\section{Bipartite entanglement with no fermion entanglement}

Previous examples show an exact correspondence between bipartite and fermion entanglement. The representations considered involve not only a fixed value of the global parity, but also of the local number parity. It is apparent, however, that it is also possible to obtain bipartite entanglement from SD's by choosing appropriate partitions of the sp space, although in this case the local parity will not be fixed. For instance, the single fermion state

$$
|\psi\rangle_{f}=\left(\alpha c_{A \uparrow}^{\dagger}+\beta c_{B \uparrow}^{\dagger}\right)|0\rangle,
$$

where the fermion is created in a state with no definite position if $\alpha \beta \neq 0$, leads obviously to $S\left(\rho^{\mathrm{sp}}\right)=0$ but corresponds to an entangled state $\alpha|\uparrow\rangle_{A} \otimes|0\rangle_{B}+\beta|0\rangle_{A} \otimes$ $|\downarrow\rangle_{B}$. However, the local states at each side have different number parity. The same occurs with the two-fermion SD $\left(\alpha c_{A \uparrow}^{\dagger}+\beta c_{B \uparrow}^{\dagger}\right)\left(\alpha^{\prime} c_{A \downarrow}^{\dagger}+\beta^{\prime} c_{B \downarrow}^{\dagger}\right)|0\rangle$, which has zero fermionic concurrence but corresponds to the entangled state $\alpha \beta^{\prime} \mid \uparrow$ \rangle$_{A} \otimes|\downarrow\rangle_{B}-\alpha^{\prime} \beta|\downarrow\rangle_{A} \otimes|\uparrow\rangle_{B}+\alpha \alpha^{\prime}|\uparrow \downarrow\rangle_{A} \otimes|0\rangle_{B}+\beta \beta^{\prime}|0\rangle_{A} \otimes$ $|\uparrow \downarrow\rangle_{B}$

Hence, although there is entanglement with respect to the $(A, B)$ partition, it is not possible to make arbitrary linear combinations of the eigenstates of $\rho_{A}$ or $\rho_{B}$, since they may not have a definite number parity. While such entanglement may be sufficient for observing Bell inequalities violation, as proposed in [30], it can exhibit limitations for other tasks involving superpositions of local eigenstates, as discussed in sec. IIII This effect will occur whenever one of the fermions is created in a state which is "split" by the chosen partition of the sp space. With the restriction of a fixed number parity at each "side" an equivalence between bipartite and fermionic entanglement can become feasible, as discussed next. Notice that such restriction directly implies blocked sp density matrices $\rho^{\mathrm{sp}}$ and $\rho^{\mathrm{qsp}}$, since all contractions $\left\langle c_{A i}^{\dagger} c_{B j}\right\rangle$ and $\left\langle c_{A i}^{\dagger} c_{B j}^{\dagger}\right\rangle$ linking both sides do not conserve the local parity and will therefore vanish $\forall i, j$. 


\section{E. Fermion entanglement as two-qubit entanglement}

Let us now return to the two-fermion state (10). The reason why the two particles become distinguishable is that the "position" observable allows us to split the sp state space $\mathcal{H}$ as the direct sum of two copies of the spin space $\mathcal{H}_{\mathcal{S}}, \mathcal{H}=\mathcal{H}_{\mathcal{S}_{A}} \oplus \mathcal{H}_{\mathcal{S}_{B}}$, with ${ }_{A}\langle\mu \mid \mu\rangle_{B}=$ $\left\langle 0\left|c_{A \mu} c_{B \mu}^{\dagger}\right| 0\right\rangle=0$ for $\mu=\uparrow$ or $\downarrow$. This last condition ensures in fact that there is just one fermion at each side $\left(N_{A(B)}|\psi\rangle_{f}=|\psi\rangle_{f}\right)$. However, for a more general twofermion state, like that considered in the previous section, it is no longer possible to perform a measurement of the spin of only one particle by coupling it with position since both particles may be found at the same site.

But now nothing prevents us from turning back the argument and state that if for an arbitrary state $|\psi\rangle_{f}$, it is possible to split $\mathcal{H}$ as $\mathcal{H}=\mathcal{H}_{A} \oplus \mathcal{H}_{B}$, where $\mathcal{H}_{A}$ and $\mathcal{H}_{B}$ contain just one fermion $\left(N_{A}|\psi\rangle_{f}=N_{B}|\psi\rangle_{f}=1\right)$, then we recover again a system of two distinguishable qubits. This last feature leads us to the following important result:

Lemma 1: Let $|\psi\rangle_{f}$ be an arbitrary pure state of a fermion system with a 4 -dimensional sp space $\mathcal{H}$, having definite number parity yet not necessarily fixed fermion number. Then the entropy (3) of the corresponding density matrix $\rho^{\mathrm{qsp}}$ is proportional to the entanglement entropy between the two distinguishable qubits that can be extracted just by measuring the appropriate observables.

Proof. We start with a general state $|\psi\rangle_{f}$ with even number parity, which in this space will have the form (7). For general $\alpha_{i j}, \alpha_{0}$ and $\alpha_{4}$ in (7), the basis of the sp space $\mathcal{H}$ determined by the fermion operators $\left\{c_{i}, c_{i}^{\dagger}\right\}$ cannot be split in order to measure only one particle at each part. This fact remains true even if $\alpha_{0}=\alpha_{4}=0$, as $\alpha$ is a general antisymmetric matrix. However, as proved in 24, it is always possible to find another basis of $\mathcal{H}$, determined by fermion operators $\left\{a_{i}, a_{i}^{\dagger}\right\}$ related to $\left\{c_{i}, c_{i}^{\dagger}\right\}$ through a Bogoliubov transformation, such that the state (7) can be rewritten as

$$
|\psi\rangle_{f}=\left(\alpha_{+} a_{1}^{\dagger} a_{2}^{\dagger}+\alpha_{-} a_{3}^{\dagger} a_{4}^{\dagger}\right)|0\rangle
$$

which is analogous to Eq. (10). Here $\left|\alpha_{ \pm}\right|^{2}=f_{ \pm}$are just the distinct eigenvalues (6) of the extended density matrix $\rho^{\mathrm{qsp}}$ determined by the state (7), whereas $\left\{a_{i}, a_{i}^{\dagger}\right\}$ are suitable quasiparticle operators diagonalizing $\rho^{\mathrm{qsp}}$. The concurrence (8) becomes $C\left(|\psi\rangle_{f}\right)=2\left|\alpha_{+} \alpha_{-}\right|$.

We then recognize (26) as the Schmidt decomposition (9) of a two-qubit state written in the fermionic representation (10), since, for instance, the sets $\left\{a_{1}^{\dagger}, a_{3}^{\dagger}\right\}$ and $\left\{a_{2}^{\dagger}, a_{4}^{\dagger}\right\}$ (analogous to $\left\{a_{A \uparrow}^{\dagger}, a_{A \downarrow}^{\dagger}\right\}$ and $\left\{a_{B \uparrow}^{\dagger}, a_{B \downarrow}^{\dagger}\right\}$ ) span subspaces $\mathcal{H}_{A}$ and $\mathcal{H}_{B}$ with $N_{A}=N_{B}=1$ $\left(N_{A(B)}|\psi\rangle_{f}=|\psi\rangle_{f}\right)$. And because the Schmidt coefficients $\left|\alpha_{ \pm}\right|^{2}$ coincide with the eigenvalues of $\rho^{\mathrm{qsp}}$, we obtain again $S\left(\rho_{A}\right)=S\left(\rho_{B}\right)=\frac{1}{4} S\left(\rho^{\mathrm{qsp}}\right)$ (Eq. (22) $)$, with the fermionic concurrence coinciding exactly with the standard one.

The case of general odd parity states, which in this sp space are linear combinations of states with one and three fermions,

$$
|\psi\rangle_{f}=\sum_{i=1}^{4} \beta_{i} c_{i}^{\dagger}|0\rangle+\tilde{\beta}_{i} c_{i}|\overline{0}\rangle,
$$

where $|\overline{0}\rangle=c_{1}^{\dagger} c_{2}^{\dagger} c_{3}^{\dagger} c_{4}^{\dagger}|0\rangle \quad$ and $\quad c_{i}|\overline{0}\rangle=$ $\frac{1}{3 !} \sum_{j, k, l} \epsilon_{i j k l} c_{j}^{\dagger} c_{k}^{\dagger} c_{l}^{\dagger}|0\rangle$, can be treated in a similar way, as they can be converted to even parity states of the form (17) by a particle-hole transformation of one of the states (i.e., $c_{1}^{\dagger} \rightarrow c_{1},|0\rangle \rightarrow c_{1}^{\dagger}|0\rangle$, leading to $\alpha_{0}=\beta_{1}, \alpha_{4}=-\tilde{\beta}_{1}, \alpha_{1 j}=-\beta_{j}$, and $\alpha_{i j}=\sum_{k} \epsilon_{i j k 1} \tilde{\beta}_{k}$ for $i, j=2,3,4$ in Eq. (17)). They can then be also written in the form (26), in terms of suitable quasiparticle operators diagonalizing $\rho^{\mathrm{qsp}}$, so that the previous considerations still hold. The concurrence of the states [27), given by 24] $C\left(|\psi\rangle_{f}\right)=2\left|\sum_{i=1}^{4} \beta_{i} \tilde{\beta}_{i}\right|$, becomes again $2\left|\alpha_{+} \alpha_{-}\right|$.

Some further comments are here in order. First, just the subspaces of $\mathcal{H}$ generated by $\left\{a_{1}^{\dagger}, a_{2}^{\dagger}\right\}$ and $\left\{a_{3}^{\dagger}, a_{4}^{\dagger}\right\}$ are defined by (26), since any unitary transformation $a_{1(2)}^{\dagger} \rightarrow \sum_{k=1,2} U_{k, 1(2)} a_{k}^{\dagger}$ (and similarly for $a_{3(4)}^{\dagger}$ ) will leave it unchanged (except for phases in $\alpha_{ \pm}$).

Secondly, we may also reinterpret the state (26) as a two-fermion state with even local number parity if side $A$ is identified with operators $\left\{a_{1}^{\dagger}, a_{2}^{\dagger}\right\}$ and $B$ with $\left\{a_{3}^{\dagger}, a_{4}^{\dagger}\right\}$, such that each side has either 0 or two fermions (even number parity qubits). Still with even local number parity we may as well rewrite it in the form (18), i.e.,

$$
|\psi\rangle_{f}=\left(\alpha_{-}+\alpha_{+} a_{1}^{\dagger} a_{3}^{\dagger} a_{2}^{\dagger} a_{4}^{\dagger}\right)|0\rangle
$$

through a transformation $a_{i}^{\dagger} \rightarrow a_{i}$ for $i=3$, 4, with $|0\rangle \rightarrow a_{3}^{\dagger} a_{4}^{\dagger}|0\rangle$. Here just the vacuum $|0\rangle$ and the completely occupied state $|\overline{0}\rangle$ are defined, since (28) remains invariant (up to a phase in $\alpha_{+}$) by any unitary transformation $a_{i}^{\dagger} \rightarrow \sum_{k} U_{k i} a_{k}^{\dagger}$ of the operators $a_{i}^{\dagger}$.

Finally, if $|\psi\rangle_{f}$ is a two-fermion state $\frac{1}{2} \sum_{i j} \alpha_{i j} c_{i}^{\dagger} c_{j}^{\dagger}|0\rangle$, the previous considerations remain valid for a sp space $\mathcal{H}$ of arbitrary dimension. In this case $\kappa=0$ and it is always possible to rewrite $|\psi\rangle_{f}$ as $[$ ]

$$
|\psi\rangle_{f}=\sum_{k} \alpha_{k} a_{k}^{\dagger} a_{\bar{k}}^{\dagger}|0\rangle
$$

where $\left|\alpha_{k}^{2}\right|$ are the eigenvalues of $\rho^{\mathrm{sp}}=\alpha \alpha^{\dagger}$ and $\left\{a_{k}, a_{\bar{k}}\right\}$ are suitable fermion operators diagonalizing this matrix, obtained through a unitary transformation $a_{k(\bar{k})}=$ $\sum_{i} \bar{U}_{i k(\bar{k})} c_{i}$ (satisfying [7] $U^{\dagger} \alpha \bar{U}=\alpha^{\prime}$ with $\alpha^{\prime}$ a block diagonal matrix with $2 \times 2$ blocks $\left.\alpha_{k}\left(\begin{array}{cc}0 & 1 \\ -1 & 0\end{array}\right)\right)$. The sp space can then be written as $\mathcal{H}_{A} \oplus \mathcal{H}_{B}$ with $\mathcal{H}_{A(B)}$ the subspaces spanned by the sets $\left\{a_{k(\bar{k})}^{\dagger}\right\}$, containing each one fermion. We thus obtain $S\left(\rho_{A}\right)=S\left(\rho_{B}\right)=\frac{1}{2} S\left(\rho^{\mathrm{sp}}\right)$ (Eq. (16) ). 


\section{F. Fermion entanglement as minimum bipartite entanglement}

We now demonstrate a second general result, concerning the mode entanglement associated with general decompositions $\mathcal{H}=\mathcal{H}_{\mathcal{A}} \oplus \mathcal{H}_{\mathcal{B}}$ of a four-dimensional sp space. Any many-fermion state can be written as $|\psi\rangle_{f}=\sum_{\mu, \nu} \alpha_{\mu \nu}|\mu \nu\rangle$, where $\mu(\nu)$ labels orthogonal SD's on $\mathcal{H}_{\mathcal{A}}\left(\mathcal{H}_{\mathcal{B}}\right)$ and $|\mu \nu\rangle=\left[\prod_{i \in \mathcal{H}_{\mathcal{A}}}\left(c_{i}^{\dagger}\right)^{n_{i}^{\mu}}\right]\left[\prod_{j \in \mathcal{H}_{\mathcal{B}}}\left(c_{j}^{\dagger}\right)^{n_{j}^{\nu}}\right]|0\rangle$ is a $\mathrm{SD}$ on $\mathcal{H}$, with $n_{i}^{\mu}=0,1$ the occupation of sp state $i$ in the state $\mu$. The ensuing reduced states $\rho_{A}=$ $\sum_{\mu, \mu^{\prime}}\left(\alpha \alpha^{\dagger}\right)_{\mu \mu^{\prime}}|\mu\rangle\left\langle\mu^{\prime}\right|$ and $\rho_{B}=\sum_{\nu, \nu^{\prime}}\left(\alpha^{T} \bar{\alpha}\right)_{\nu \nu^{\prime}}|\nu\rangle\left\langle\nu^{\prime}\right|$ satisfy $\operatorname{Tr} \rho_{A(B)} O_{A(B)}={ }_{f}\left\langle\psi\left|O_{A(B)}\right| \psi\right\rangle_{f}$ for any operator depending just on the local fermions $\left\{c_{i}, c_{i}^{\dagger}, i \in \mathcal{H}_{A(B)}\right\}$. The entanglement entropy associated with such bipartition is then 26] $E(A, B)=S\left(\rho_{A}\right)=S\left(\rho_{B}\right)$.

In the present case we may have either $2+2$ bipartitions $\left(\operatorname{dim} \mathcal{H}_{\mathcal{A}}=\operatorname{dim} \mathcal{H}_{\mathcal{B}}=2\right.$ ), or $1+3$ bipartitions $\left(\operatorname{dim} \mathcal{H}_{\mathcal{A}}=1, \operatorname{dim} \mathcal{H}_{\mathcal{B}}=3\right)$. In the latter the entanglement is determined just by the average occupation of the single state of $\mathcal{H}_{\mathcal{A}}$ 24] and corresponds to the case where $A$ has access to just one of the sp states possibly occupied in $|\psi\rangle_{f}$. A realization of a $2+2$ partition is just that of spin 1/2 fermions which can be at two-different sites (one accessible to Alice and the other to Bob), while a $1+3$ bipartition could be one where Alice has access to one site and just one spin direction, i.e., to the knowledge of the occupation of the sp state $A_{\uparrow}$. It could also apply to any asymmetric situation like that where spins are all up (i.e., aligned along the field direction) but the fermions can be in four different locations or orbital states, with only one accessible to Alice.

Lemma 2: Let $|\psi\rangle_{f}$ be a general definite number parity fermion state in a sp space $\mathcal{H}$ of dimension 4 , and let $\mathcal{H}=$ $\mathcal{H}_{A} \oplus \mathcal{H}_{B}$ be an arbitrary decomposition of $\mathcal{H}$ with $\mathcal{H}_{A}$ and $\mathcal{H}_{B}$ of finite dimension. The entanglement entropy associated with such bipartition satisfies

$$
S\left(\rho_{A}\right)=S\left(\rho_{B}\right) \geq \frac{1}{4} S\left(\rho^{\mathrm{qsp}}\right) .
$$

Eq. (29) holds for any entropic form $S(\rho)=\operatorname{Tr} f(\rho)(f$ concave, $f(0)=f(1)=0)$.

Hence, the fermionic entanglement represents the minimum bipartite entanglement that can be obtained in such a space, which is reached for those bipartitions arising from the normal forms (26) or (28). The greater entanglement in a $2+2$ bipartition is obtained at the expense of loosing a fixed number parity in the local reduced states. Note that $S\left(\rho^{\mathrm{qsp}}\right)$ vanishes only if $|\psi\rangle_{f}$ is a quasiparticle vacuum or SD in some sp basis, while $S\left(\rho_{A(B)}\right)$ does so only when the previous condition holds in a basis compatible with the chosen bipartition.

We will actually show the equivalent majorization 34. relation

$$
\lambda\left(\rho_{A(B)}\right) \prec\left(f_{+}, f_{-}\right),
$$

where $\lambda\left(\rho_{A(B)}\right)$ denotes the spectrum of $\rho_{A}$ or $\rho_{B}$ sorted in decreasing order and $f_{+}, f_{-}=1-f_{+} \leq f_{+}$are the distinct eigenvalues (6) (fourfold degenerate) of $\rho^{\mathrm{qsp}}$. Eq. (30) is then equivalent to the condition $\lambda_{\max } \leq f_{+}$, with $\lambda_{\max }$ the largest eigenvalue of $\rho_{A(B)}$, and implies (29), while (29) implies (30) if valid for any entropic function $f$ 3.5.

Proof. Consider first a general even parity state (7) and a $2+2$ decomposition $\mathcal{H}=\mathcal{H}_{A} \oplus \mathcal{H}_{B}$, with $\mathcal{H}_{A} \equiv \mathcal{H}_{12}$, $\mathcal{H}_{B} \equiv \mathcal{H}_{34}$ and $\mathcal{H}_{i j}$ the subspace generated by $\left\{c_{i}^{\dagger}, c_{j}^{\dagger}\right\}$. Changing to the notation $A_{1}, A_{2}, B_{1}, B_{2}$ for sp states $1,2,3,4$, we can rewrite (7) as a sum of states of the form (14) and (20) (Fig. 11):

$|\psi\rangle_{f}=\sum_{\mu, \nu} \beta_{\mu \nu} c_{A_{\mu}}^{\dagger} c_{B_{\nu}}^{\dagger}|0\rangle+\sum_{\mu, \nu} \tilde{\beta}_{\mu \nu}\left(c_{A_{1}}^{\dagger} c_{A_{2}}^{\dagger}\right)^{n_{\mu}}\left(c_{B_{1}}^{\dagger} c_{B_{2}}^{\dagger}\right)^{n_{\nu}}|0\rangle$,

where $\mu, \nu=1,2, \beta_{\mu \nu}=\alpha_{\mu, \nu+2}, n_{\mu}=\mu-1, \tilde{\beta}_{11}=\alpha_{0}$, $\tilde{\beta}_{22}=\alpha_{4}, \tilde{\beta}_{12}=\alpha_{34}$, and $\tilde{\beta}_{21}=\alpha_{12}$. The first (second) sum in (31) is the odd (even) local number parity component.

After local unitary transformations $c_{S \mu} \rightarrow \sum_{\nu} \bar{U}_{\nu \mu}^{S} c_{S \nu}$, $S=A, B$, which will not affect the vacuum nor the even local parity component (except for phases in $\tilde{\beta}_{\mu \nu}$, determined by $\operatorname{det} U^{S}$ ), we can set $\beta_{\mu \nu}$ diagonal. Similarly, after local Bogoliubov transformations $c_{S_{1}} \rightarrow u_{S} c_{S_{1}}+$ $v_{S} c_{S_{2}}^{\dagger}, c_{S_{2}} \rightarrow u_{S} c_{S_{2}}-v_{S} c_{S_{1}}^{\dagger},\left|u_{S}^{2}\right|+\left|v_{S}^{2}\right|=1$, with $|0\rangle \rightarrow\left[\prod_{S=A, B}\left(u_{S}-v_{S} c_{S_{1}}^{\dagger} c_{S_{2}}^{\dagger}\right)\right]|0\rangle$, we can set $\tilde{\beta}_{\mu \nu}$ diagonal as discussed in sec. IIC Though modifying the vacuum, they will not change the form of the odd local parity component except for phases in $\beta_{\mu \nu}$. Thus, by local transformations it is possible to rewrite (31) as

$|\psi\rangle_{f}=\left(\beta_{1} c_{A_{1}}^{\dagger} c_{B_{1}}^{\dagger}+\beta_{2} c_{A_{2}}^{\dagger} c_{B_{2}}^{\dagger}+\tilde{\beta}_{1}+\tilde{\beta}_{2} c_{A_{1}}^{\dagger} c_{A_{2}}^{\dagger} c_{B_{1}}^{\dagger} c_{B_{2}}^{\dagger}\right)|0\rangle$,

where $\left|\beta_{\mu}\right|$ and $\left|\tilde{\beta}_{\mu}\right|$ are the singular values of the $2 \times 2$ matrices $\beta$ and $\tilde{\beta}$ in (31). Eq. (32) is the Schmidt decomposition for this partition, with $\left(\left|\beta_{1}^{2}\right|,\left|\beta_{2}^{2}\right|,\left|\tilde{\beta}_{1}^{2}\right|,\left|\tilde{\beta}_{2}^{2}\right|\right)$ the eigenvalues of the reduced density matrices $\rho_{A}$ and $\rho_{B}$ of modes $\left(A_{1}, A_{2}\right)$ and $\left(B_{1}, B_{2}\right)$ respectively.

Now, suppose $\lambda_{\max }=\left|\beta_{1}^{2}\right|$. We have

$$
\left|\beta_{1}\right|^{2} \leq\left|\beta_{1}\right|^{2}+\left|\tilde{\beta}_{2}\right|^{2}=\left\langle c_{A_{1}}^{\dagger} c_{A_{1}}\right\rangle .
$$

But $\left\langle c_{A_{1}}^{\dagger} c_{A_{1}}\right\rangle=\sum_{k=1}^{8}\left|W_{A_{1}, k}\right|^{2} f_{k}$, where $f_{k}$ are the eigenvalues of $\rho^{\text {qsp }}$ (equal to $f_{+}$or $f_{-}$) and $W$ the unitary matrix diagonalizing $\rho^{\mathrm{qsp}}\left(\sum_{k=1}^{8}\left|W_{A_{1}, k}\right|^{2}=1\right)$. Therefore,

$$
f_{-} \leq\left\langle c_{A_{1}}^{\dagger} c_{A_{1}}\right\rangle \leq f_{+} \text {. }
$$

Eqs. (33)- (34) imply $\left|\beta_{1}\right|^{2} \leq f_{+}$, which demonstrates Eq. (30) and hence (29) for a general $2+2$ bipartition $\mathcal{H}_{A} \oplus \mathcal{H}_{B}$. For $\lambda_{\max }$ equal to any other coefficient the proof is similar.

Moreover, Eq. (34) also shows that the sorted spectrum $\lambda\left(\rho_{A_{1}\left(A_{2}, B\right)}\right)=\left(\left\langle c_{A_{1}}^{\dagger} c_{A_{1}}\right\rangle, 1-\left\langle c_{A_{1}}^{\dagger} c_{A_{1}}\right\rangle\right)^{\downarrow}$ associated with the $1+3$ bipartition $\mathcal{H}_{A_{1}} \oplus \mathcal{H}_{A_{2}, B}$ satisfies $\lambda\left(\rho_{A_{1},\left(A_{2}, B\right)}\right) \prec\left(f_{+}, f_{-}\right)$. In the latter $S\left(\rho_{A_{1}}\right)$ is the 
entanglement between the sp mode $A_{1}$ and its orthogonal complement as defined in 24, 26], determined by the average occupation $\left\langle c_{A_{1}}^{\dagger} c_{A_{1}}\right\rangle$ of the mode. Hence, Eqs. (29)-(30) hold as well for any $1+3$ bipartition.

And equality in (29) for all entropic functions is evidently reached only for those bipartitions arising from the normal forms (26)-(28): Considering the non-trivial case $f_{+}<1$, if equality in (29) is to hold for all entropies, necessarily $\rho_{A(B)}$ should be of rank 2 with $\lambda\left(\rho_{A(B)}\right)=$ $\left(f_{+}, f_{-}\right)$. For a $1+3$ bipartition, this identity directly implies $\left\langle c_{A_{1}}^{\dagger} c_{A_{1}}\right\rangle=f_{+}$or $f_{-}$and hence a bipartition arising from a normal form (26)-28), where $A \equiv A_{1}$ is one of the sp states of the normal basis. And for a $2+2$ bipartition, it implies that the two eigenstates of $\rho_{A}$ with non-zero eigenvalues $f_{ \pm}$should have the same number parity, since otherwise Eq. (8) would imply $C\left(|\psi\rangle_{f}\right)=0$ and therefore $f_{+}=1$, in contrast with the assumption. Hence such bipartition must arise from a normal form (26) or (28).

The demonstration of previous results for odd global number parity states is similar, as they can be rewritten as even parity states after a particle-hole transformation.

Some further comments are also in order. We may rewrite the state (32) as

$$
|\psi\rangle_{f}=\sqrt{p_{-}}\left|\psi_{-}\right\rangle_{f}+\sqrt{p_{+}}\left|\psi_{+}\right\rangle_{f},
$$

where $\left|\psi_{-}\right\rangle_{f}=\frac{1}{\sqrt{p_{-}}}\left(\beta_{1} c_{A_{1}}^{\dagger} c_{B_{1}}^{\dagger}+\beta_{2} c_{A_{2}}^{\dagger} c_{B_{2}}^{\dagger}\right)|0\rangle,\left|\psi_{+}\right\rangle_{f}=$ $\frac{1}{\sqrt{p_{+}}}\left(\tilde{\beta}_{1}+\tilde{\beta}_{2} c_{A_{1}}^{\dagger} c_{A_{2}}^{\dagger} c_{B_{1}}^{\dagger} c_{B_{2}}^{\dagger}\right)|0\rangle$ are the normalized odd and even local parity components and $p_{-}=\left|\beta_{1}^{2}\right|+\left|\beta_{2}^{2}\right|$, $p_{+}=\left|\tilde{\beta}_{1}^{2}\right|+\left|\tilde{\beta}_{2}^{2}\right|=1-p_{-}$. We then see that for the von Neumann entropy, we obtain

$$
S\left(\rho_{A}\right)=S\left(\rho_{B}\right)=p_{-} S\left(\rho_{A}^{-}\right)+p_{+} S\left(\rho_{A}^{+}\right)+S(p),
$$

where the first two terms represent the average of the entanglement entropies of the odd and even local parity components $\left(S\left(\rho_{A}^{-}\right)=-\sum_{\mu} \frac{\left|\beta_{\mu}^{2}\right|}{p_{-}} \log _{2} \frac{\left|\beta_{\mu}^{2}\right|}{p_{-}}, S\left(\rho_{A}^{+}\right)=\right.$ $\left.-\sum_{\mu} \frac{\left|\tilde{\beta}_{\mu}^{2}\right|}{p_{+}} \log _{2} \frac{\left|\tilde{\beta}_{\mu}^{2}\right|}{p_{+}}\right)$while $S(p)=-\sum_{\nu= \pm} p_{\nu} \log _{2} p_{\nu}$ is the additional entropy arising from the mixture of both local parities. We then have $0 \leq S\left(\rho_{A}\right) \leq 2$, with the maximum $S\left(\rho_{A}\right)=2$ reached iff $S\left(\rho_{A}^{ \pm}\right)=1$ and $p_{ \pm}=\frac{1}{2}$.

On the other hand, the fermionic concurrence (8) of the state (32) is just

$$
C\left(|\psi\rangle_{f}\right)=2\left|\beta_{1} \beta_{2}+\tilde{\beta}_{1} \tilde{\beta}_{2}\right| .
$$

It then satisfies

$$
\left|p_{-} C_{-}-p_{+} C_{+}\right| \leq C\left(|\psi\rangle_{f}\right) \leq p_{-} C_{-}+p_{+} C_{+},
$$

where $C_{ \pm}=C\left(\left|\psi_{ \pm}\right\rangle_{f}\right)=2\left(\begin{array}{l}\left|\tilde{\beta}_{1} \tilde{\beta}_{2}\right| / p_{+} \\ \left|\beta_{1} \beta_{2}\right| / p_{-}\end{array}\right)$are the concurrences of the even and odd local parity components. We then see, for instance, that for maximum bipartite entanglement $S\left(\rho_{A}\right)=2, C_{ \pm}=1$ and hence $C\left(|\psi\rangle_{f}\right)$ can
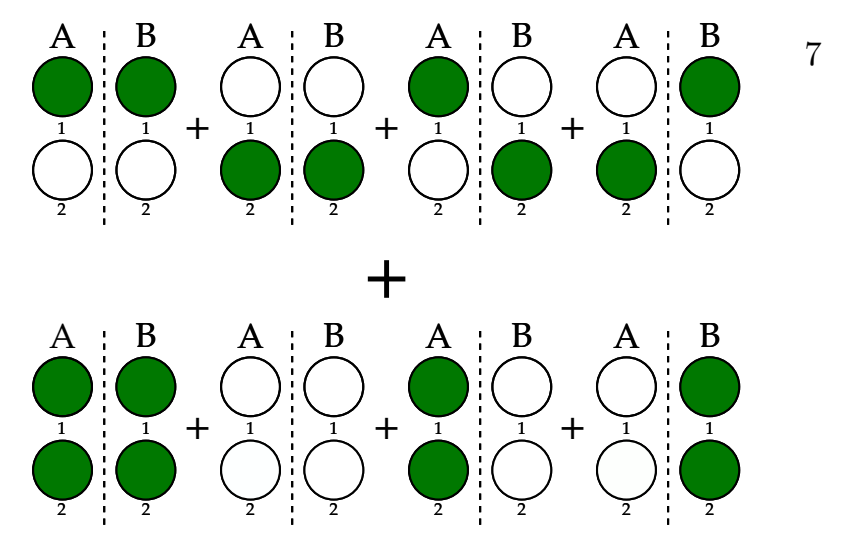

FIG. 1. Depiction of the eight even number parity fermion states of four single-particle modes, partitioned such that the two modes on the left of the dashed line belong to Alice, whereas the two modes on the right to Bob. The upper row are the states with odd local number parity (one fermion for Alice and one fermion for Bob) whereas the bottom row those with even local number parity (Alice and Bob may have 0 or two fermions). The states of the bottom row can be formally obtained from those on the top by performing particle-hole transformations $c_{A 2}^{\dagger} \leftrightarrow c_{A 2}$ and $c_{B 2}^{\dagger} \leftrightarrow c_{B 2}$.

take any value between 0 and 1 , according to the relative phase between the even and odd local parity components.

Finally, it is obviously possible to rewrite the Schmidt form (32) as a two-fermion state by means of suitable local particle-hole transformations (i.e. $c_{B_{\mu}} \rightarrow c_{B_{\mu}}^{\dagger}, \mu=$ 1,2 , with $\left.|0\rangle \rightarrow c_{B_{1}}^{\dagger} c_{B_{2}}^{\dagger}|0\rangle\right)$. After some relabelling, we obtain the equivalent form

$|\psi\rangle_{f}=\left(\beta_{1} c_{A_{1}}^{\dagger} c_{B_{1}}^{\dagger}+\beta_{2} c_{A_{2}}^{\dagger} c_{B_{2}}^{\dagger}+\tilde{\beta}_{2} c_{A_{1}}^{\dagger} c_{A_{2}}^{\dagger}-\tilde{\beta}_{1} c_{B_{1}}^{\dagger} c_{B_{2}}^{\dagger}\right)|0\rangle$,

where terms with two fermions at the same side side are added to the form (10). Therefore, all previous considerations (35)-38) can be realized with a fixed total number of fermions, with expression (37) still valid.

\section{APPLICATION}

The formalism of the previous sections may now be used to rewrite a qubit-based quantum circuit as a circuit based on fermionic modes. It is easy to see by now that any pair of fermionic modes, say $i, j$, prepared in such a way that their total occupation is constrained to $N_{i j}=c_{i}^{\dagger} c_{i}+c_{j}^{\dagger} c_{j}=1$, is essentially a qubit. Therefore, a collection of $n$ such pairs of modes constitutes a system of $n$ qubits. Furthermore any single-qubit operation can be performed on each pair just by using unitaries in $\mathcal{H}$ linking only these two modes, and these unitaries can be always written in terms of the effective Pauli operators (11), i.e., $\sigma_{x}^{i j}=c_{i}^{\dagger} c_{j}+c_{j}^{\dagger} c_{i}$, $\sigma_{y}^{i j}=i\left(c_{j}^{\dagger} c_{i}-c_{i}^{\dagger} c_{j}\right), \sigma_{z}^{i j}=c_{i}^{\dagger} c_{i}-c_{j}^{\dagger} c_{j}$. The last ingredient for universal computation is the CNOT gate, which in the tensor product space $A \otimes B$ can be written as $U_{\mathrm{CNOT}}=|0\rangle\langle 0|\otimes I+| 1\rangle\langle 1| \otimes \sigma_{x}=\exp \left[i \frac{\pi}{4}\left(1-\sigma_{z}\right) \otimes\left(1-\sigma_{x}\right)\right]$. In the fermionic representation, if $A$ is spanned by modes 


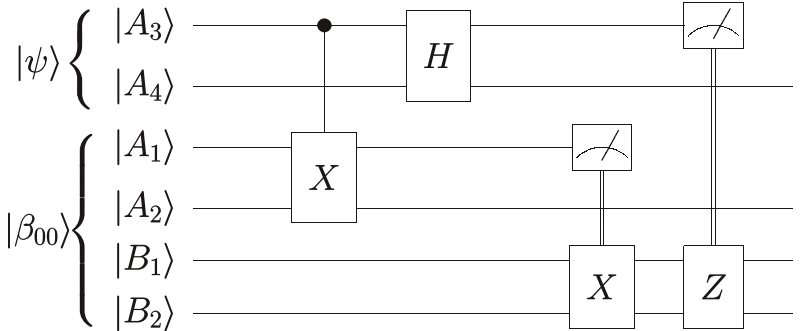

FIG. 2. Teleportation protocol with the present fermionic implementation. Each qubit is represented by a pair of fermionic modes having a total occupation number of 1 . The control operation can be realized involving just one of the modes of the pair representing the control qubit due to the occupation number constraint, and similarly the usual measurement in the standard basis can be implemented by measuring just one of these modes. If the pair occupation number constraint is relaxed so that both local number parities coexist, then control and measurement operations involve both modes.

$i j$ and $B$ by the different modes $k l$, for states having one fermion at each pair of modes it can be written as

$$
U_{\mathrm{CNOT}}^{f}=\exp \left[i \frac{\pi}{4}\left(1-\sigma_{z}^{i j}\right)\left(1-\sigma_{x}^{k l}\right)\right] .
$$

Since just an even number of fermion operators $c$ per pair are involved, its action is not affected by the state of intermediate pairs. It is then possible to implement any qubit-based quantum circuit using fermion states.

As an example, in Fig. 2 we show the teleportation protocol adapted to be implemented using an entangled fermion state as resource, and a two mode sate to be teleported. Alice has the modes $\left\{\left|A_{1}\right\rangle,\left|A_{2}\right\rangle,\left|A_{3}\right\rangle,\left|A_{4}\right\rangle\right\}$ while Bob is in possession of $\left\{\left|B_{1}\right\rangle,\left|B_{2}\right\rangle\right\}$. The first two modes of Alice are entangled with those of Bob, being in the joint state $\left|\beta_{00}\right\rangle=\frac{1}{\sqrt{2}}\left(c_{A_{1}}^{\dagger} c_{B_{1}}^{\dagger}+c_{A_{2}}^{\dagger} c_{B_{2}}^{\dagger}\right)|0\rangle$, and the remaining modes of Alice are in the state $|\psi\rangle=\left(\alpha c_{A_{3}}^{\dagger}+\right.$ $\left.\beta c_{A_{4}}^{\dagger}\right)|0\rangle,|\alpha|^{2}+\left|\beta^{2}\right|=1$. The input state is therefore

$$
\left|\psi_{i}\right\rangle=\frac{1}{\sqrt{2}}\left(\alpha c_{A_{3}}^{\dagger}+\beta c_{A_{4}}^{\dagger}\right)\left(c_{A_{1}}^{\dagger} c_{B_{1}}^{\dagger}+c_{A_{2}}^{\dagger} c_{B_{2}}^{\dagger}\right)|0\rangle
$$

and it is straightforward to see that the output state is

$$
\begin{aligned}
\left|\psi_{o}\right\rangle= & \frac{1}{2}\left[c_{A_{4}}^{\dagger} c_{A_{2}}^{\dagger}\left(\alpha c_{B_{1}}^{\dagger}+\beta c_{B_{2}}^{\dagger}\right)+c_{A_{4}}^{\dagger} c_{A_{1}}^{\dagger}\left(\alpha c_{B_{2}}^{\dagger}+\beta c_{B_{1}}^{\dagger}\right)+\right. \\
& \left.c_{A_{3}}^{\dagger} c_{A_{2}}^{\dagger}\left(-\alpha c_{B_{1}}^{\dagger}+\beta c_{B_{2}}^{\dagger}\right)+c_{A_{3}}^{\dagger} c_{A_{1}}^{\dagger}\left(-\alpha c_{B_{2}}^{\dagger}+\beta c_{B_{1}}^{\dagger}\right)\right]|0\rangle
\end{aligned}
$$

The controlled operations on Bob's modes depicted in Fig. 2 then ensure that his output will be the state $|\psi\rangle$.

Considering now a general circuit, if the input states are restricted to be SD's in the previous basis, with one fermion for each pair, we recover a classical circuit. The CNOT gate in (40) reduces for these states to a classical controlled swap or Fredkin gate, which implies that reversible classical computation can be done with SD's as input states.

On the other hand, if the occupation number restriction $N_{i j}=1$ (i.e., odd number parity for each pair) is relaxed, so that the building blocks of the circuit are no longer single fermions that can be found in two possible states, but rather the fermionic modes themselves, other possibilities arise. For instance, if now the input states contain either 0 or two fermions for each pair (even number parity qubits), such that modes $i, j$ are either both empty or both occupied, then we should use the $\tilde{\sigma}_{\mu}^{i j}$ operators as defined in (19), i.e., $\tilde{\sigma}_{x}^{i j}=c_{i}^{\dagger} c_{j}^{\dagger}+c_{j} c_{i}$, $\tilde{\sigma}_{y}^{i j}=i\left(c_{j} c_{i}-c_{i}^{\dagger} c_{j}^{\dagger}\right), \tilde{\sigma}_{z}^{i j}=c_{i}^{\dagger} c_{i}+c_{j}^{\dagger} c_{j}-1$. In this case the operator $\tilde{U}_{\text {CNOT }}^{f}$ should be constructed as in Eq. (40) with the $\tilde{\sigma}_{\mu}$ operators, while the operator (40), and in fact any unitary gate built with the $\sigma_{\mu}^{i j}$ operators, will become an identity for these states, as previously stated. Hence, by adding the appropriate gates, the same modes can in principle be used for even and odd number parity qubits independently.

For example, in the even local parity setting the input state for the teleportation protocol would be

$$
\left|\tilde{\psi}_{i}\right\rangle=\frac{1}{\sqrt{2}}\left(\beta+\alpha c_{A_{3}}^{\dagger} c_{A_{4}}^{\dagger}\right)\left(1+c_{A_{1}}^{\dagger} c_{A_{2}}^{\dagger} c_{B_{1}}^{\dagger} c_{B_{2}}^{\dagger}\right)|0\rangle .
$$

If $|0\rangle$ stands for a reference SD (Fermi sea), then this state involves 0 , one, two and three particle hole excitations, with $A_{4}, A_{2}, B_{2}$, standing for holes. The output state becomes

$$
\begin{aligned}
\left|\tilde{\psi}_{o}\right\rangle= & \frac{1}{2}\left[\left(\beta+\alpha c_{B_{1}}^{\dagger} c_{B_{2}}^{\dagger}\right)+c_{A_{1}}^{\dagger} c_{A_{2}}^{\dagger}\left(\alpha+\beta c_{B_{1}}^{\dagger} c_{B_{2}}^{\dagger}\right)+c_{A_{3}}^{\dagger} c_{A_{4}}^{\dagger} \times\right. \\
& \left.\left(\beta-\alpha c_{B_{1}}^{\dagger} c_{B_{2}}^{\dagger}\right)+c_{A_{1}}^{\dagger} c_{A_{2}}^{\dagger} c_{A_{3}}^{\dagger} c_{A_{4}}^{\dagger}\left(-\alpha+\beta c_{B_{1}}^{\dagger} c_{B_{2}}^{\dagger}\right)\right]|0\rangle,
\end{aligned}
$$

so that if Alice measures which of her modes are occupied and sends the result to Bob, he can reconstruct the original state by applying the pertinent $\tilde{X} \equiv i e^{-i \frac{\pi}{2} \tilde{\sigma}_{x}^{12}}$ and $\tilde{Z} \equiv i e^{-i \frac{\pi}{2} \tilde{\sigma}_{z}^{12}}$ operators.

Finally, let us consider the case of superdense coding [4, 36]. It is clear from the previous discussion that it can be implemented with the fermionic $\left|\beta_{00}\right\rangle$ state of the teleportation example and performing exactly the same local operations of the usual case, but viewed now as two-mode operations. Now a general state with even global parity of the four modes $\left\{\left|A_{1}\right\rangle,\left|A_{2}\right\rangle,\left|B_{1}\right\rangle,\left|B_{2}\right\rangle\right\}$ is a combination of eight states as in Eq. (7): six two-particle states, the vacuum $|0\rangle$ and the completely occupied state $|\overline{0}\rangle$, as shown in Fig. 1 Four of the six two-particle states (top of Fig. 团, have $N_{A}=N_{B}=1$ and can be used to reproduce the known results of the standard protocol. But the four remaining states, which have even local parity, may be used as well for superdense coding if the proper local operations expressed in terms of the $\tilde{\sigma}_{\mu}^{A B}$ are performed.

A general even parity state (7) may then be thought of as a superposition of states of two different two-qubit systems, like in Eqs. (31) and (35). Defining the maximally entangled orthogonal definite local parity states

$$
\begin{aligned}
& \left|\beta_{00}\right\rangle=\gamma\left(c_{A_{1}}^{\dagger} c_{B_{1}}^{\dagger} \pm c_{A_{2}}^{\dagger} c_{B_{2}}^{\dagger}\right)|0\rangle,\left|\tilde{\beta}_{00}\right\rangle=\gamma\left(c_{A_{1}}^{\dagger} c_{A_{2}}^{\dagger} c_{B_{1}}^{\dagger} c_{B_{2}}^{\dagger} \pm 1\right)|0\rangle \\
& \left|\beta_{01}\right\rangle=\gamma\left(c_{A_{1}}^{\dagger} c_{B_{2}}^{\dagger} \pm c_{A_{2}}^{\dagger} c_{B_{1}}^{\dagger}\right)|0\rangle,\left|\tilde{\beta}_{11}\right\rangle=\gamma\left(c_{A_{1}}^{\dagger} c_{A_{2}}^{\dagger} \pm c_{B_{1}}^{\dagger} c_{B_{2}}^{\dagger}\right)|0\rangle
\end{aligned}
$$

with $\gamma=\frac{1}{\sqrt{2}}$, we may consider for instance the state

$$
\left|\Psi_{00}\right\rangle=\frac{1}{\sqrt{2}}\left(\left|\beta_{00}\right\rangle+\left|\tilde{\beta}_{00}\right\rangle\right) .
$$


By implementing on (41) the identity and the local operations $i e^{-i \frac{\pi}{2}\left(\sigma_{\mu}^{A}+\tilde{\sigma}_{\mu}^{A}\right)}=\sigma_{\mu}+\tilde{\sigma}_{\mu}, \mu=x, y, z$, and taking into account Eq. (24), Alice can generate four orthogonal states: $\left|\Psi_{00}\right\rangle$ and

$$
\begin{aligned}
& \left|\Psi_{01}\right\rangle=i e^{-i \frac{\pi}{2}\left(\sigma_{x}^{A}+\tilde{\sigma}_{x}^{A}\right)}\left|\Psi_{00}\right\rangle=\frac{1}{\sqrt{2}}\left(\left|\beta_{01}\right\rangle+\left|\tilde{\beta}_{01}\right\rangle\right), \\
& \left|\Psi_{10}\right\rangle=i e^{-i \frac{\pi}{2}\left(\sigma_{z}^{A}+\tilde{\sigma}_{z}^{A}\right)}\left|\Psi_{00}\right\rangle=\frac{1}{\sqrt{2}}\left(\left|\beta_{10}\right\rangle+\left|\tilde{\beta}_{10}\right\rangle\right) \\
& \left|\Psi_{11}\right\rangle=-e^{-i \frac{\pi}{2}\left(\sigma_{y}^{A}+\tilde{\sigma}_{y}^{A}\right)}\left|\Psi_{00}\right\rangle=\frac{1}{\sqrt{2}}\left(\left|\beta_{11}\right\rangle+\left|\tilde{\beta}_{11}\right\rangle\right) .
\end{aligned}
$$

But she can also perform these operations with a local parity gate $P^{A}=-\exp \left[i \pi N_{A}\right]$ that changes the sign of local even parity states. This allows her to locally generate another set of four orthogonal states,

$$
\left|\tilde{\Psi}_{i j}\right\rangle=P^{A}\left|\Psi_{i j}\right\rangle=\frac{1}{\sqrt{2}}\left(\left|\beta_{i j}\right\rangle-\left|\tilde{\beta}_{i j}\right\rangle\right), \quad i, j=0,1,
$$

which are orthogonal to each other and to the states (41)(42). Hence, by relaxing the occupation number constraint on the partitions it is possible for Alice to send 8 orthogonal states to Bob, i.e. three bits of information, using only two modes and local unitary operations that preserve the local parity, while with one type of qubits and the same operations she can send only two bits. Of course, if parity restrictions were absent and she could change the local (and hence the global) parity she could send four bits (in agreement with the maximum capacity for two $d=4$ qudits, which is $\log _{2} d^{2}[36]$ ). A fixed global parity constraint reduces the total number of orthogonal states she can send to Bob by half.

On the other hand, since the state (41) does not have a definite local number parity, the ensuing bipartite entanglement is not restricted by the fermionic entanglement as shown in sec. IIF In fact all previous 8 states (41), (42) and (43) have maximum bipartite entanglememt, leading to maximally mixed reduced states $\rho_{A(B)}$ : $S\left(\rho_{A}\right)=S\left(\rho_{B}\right)=2$, while by applying Eq. (8) it is seen that the fermionic concurrence of the previous states is $C\left(\left|\Psi_{i j}\right\rangle\right)=C\left(\left|\tilde{\Psi}_{i j}\right\rangle\right)=1$. The unitary operations applied by Alice are local and hence cannot change the bipartite entanglement, while they are also one-body unitaries (i.e., exponents of quadratic fermion operators) so that they cannot change the fermionic concurrence and entanglement (i.e., the eigenvalues of $\rho^{\mathrm{qsp}}$ ) either. In fact, the fermionic entanglement is here not required. By changing the seed state (i.e., $\left|\Psi_{00}^{\prime}\right\rangle=\frac{1}{\sqrt{2}}\left(\left|\beta_{00}\right\rangle+\left|\tilde{\beta}_{10}\right\rangle\right)$ ), it is possible for Alice to generate locally 8 orthogonal states with the same bipartite entanglement yet no fermion entanglement $\left(C\left(\left|\Psi_{00}^{\prime}\right\rangle\right)=0\right)$.

Therefore the entanglement built with local states with different number parity plays the role of a resource for superdense coding. In fact even the state 25) with $\alpha=\beta=\frac{1}{\sqrt{2}}$, which has obviously null concurrence, can in principle be used for sending two bits if Alice can perform the parity preserving operations $P^{A}=-\exp \left[i \pi N_{A}\right]$, $\sigma_{x}+\tilde{\sigma}_{x}$ and $P^{A}\left(\sigma_{x}+\tilde{\sigma}_{x}\right)$. It is worth noting, however, that the same state cannot be directly used as a resource for teleportation with the standard protocol without violating the parity superselection rule, since Bob's two local states have opposite parity and cannot be superposed. After a measurement of Alice's modes Bob's reduced state will collapse to a state of definite parity in a realizable protocol, so that it will be impossible for him to recover a general state $|\psi\rangle$.

We have so far considered just the number parity restriction. If other superselection rules (like charge or fermion number) also apply for a particular realization they will imply stronger limitations on the capacity of states like (41). Nonetheless, even local parity qubits with no fixed fermion number remain realizable through particle-hole realizations, i.e., excitations over a reference Fermi sea in a many-fermion system.

We also mention that a basic realization of four dimensional sp space-based fermionic qubits is that of a pair of spin $1 / 2$ fermions in the two lowest states of a double well scenario in a magnetic field, which would control the energy gap between both spin directions and the transitions between them. For single occupation of each well we would have odd local parity qubits, while allowing double or zero occupancy through hopping between wells we could also have even local parity qubits.

\section{CONCLUSIONS}

We have first shown that there is an exact correspondence between bipartite states and two-fermion states of the form (14) having a fixed local number parity. Entangled states are represented by fermionic states which are not Slater Determinants, and reduced local states correspond to blocks of the sp density matrix. In particular, qubits can be represented by pairs of fermionic modes with occupation number restricted to 1 (odd number parity qubits). This result allows to rewrite qubitbased quantum circuits as fermionic circuits. But in addition, a fermionic system also enables zero or double occupancy of these pairs, which gives rise to a second type of qubit (even number parity qubits). Dual type circuits can then be devised, as the gates for one parity become identities for the other parity. And even though both types of qubits cannot be locally superposed due to the parity superselection rule, they can contribute to the entanglement in a global fixed parity state.

We have then demonstrated rigorous properties of the basic but fundamental case of a four-dmensional sp space. First, there is always a single-particle (or quasiparticle) basis in which any pure state can be seen as a state of two distinguishable qubits, with the fermionic concurrence determining the entanglement between these two qubits (Eq. (22)). Such entanglement is "genuine", in the sense that the local states involved have a definite parity and can therefore be combined. Secondly, such fermionic entanglement was shown to provide always a lower bound to the entanglement obtained with any other bipartition of this sp space, although the extra entanglement arises from the superposition of states with different local parity. While its capacity for protocols involving superpo- 
sitions of local states is limited, such entanglement can nevertheless still be useful for other tasks such as superdense coding.

\section{ACKNOWLEDGMENTS}

The authors acknowledge support from CONICET (N.G.) and CIC (R.R.) of Argentina. Work supported by CIC and CONICET PIP 112201501-00732.
[1] L. Amico, R. Fazio, A. Osterloh, V. Vedral, Rev. Mod. Phys. 80, 517 (2008).

[2] R. Horodecki, P. Horodecki, M. Horodecki, K. Horodecki, Rev. Mod. Phys. 81, 865 (2009).

[3] J. Eisert, M. Cramer, M.B. Plenio, Rev. Mod. Phys. 82, 277 (2010).

[4] M.A. Nielsen and I.L. Chuang, Quantum Computation and Quantum Information (Cambridge Univ. Press, Cambridge, UK, 2000).

[5] C.H. Bennett, G. Brassard, C. Crepeau, R. Jozsa, A. Peres, W.K. Wootters, Phys. Rev. Lett. 70, 1895 (1993).

[6] R. Josza and N. Linden, Proc. R. Soc. London, Sec. A 459, 2011 (2003); G. Vidal, Phys. Rev. Lett. 91, 147902 (2003).

[7] J. Schliemann, J.I. Cirac, M. Kus, M. Lewenstein, D. Loss, Phys. Rev. A 64, 022303 (2001).

[8] J. Schliemann, D. Loss, and A.H. MacDonald, Phys. Rev. B 63, 085311 (2001).

[9] K. Eckert, J. Schliemann, D. Bruß and M. Lewenstein, Ann. Phys. 299, 88 (2002).

[10] H.M. Wiseman and J.A. Vaccaro, Phys. Rev. Lett. 91, 097902 (2003).

[11] G.C. Ghirardi and L. Marinatto Phys. Rev. A 70, 012109 (2004).

[12] C.V. Kraus, M.M. Wolf, J.I. Cirac, G. Giedke, Phys. Rev. A 79, 012306 (2009).

[13] P. Zanardi, Phys. Rev. A 65, 042101 (2002).

[14] Y. Shi, Phys. Rev. A 67, 024301 (2003).

[15] N. Friis, A.R. Lee, and D.E. Bruschi, Phys. Rev. A 87, 022338 (2013).

[16] F. Benatti, R. Floreanini, U. Marzolino, Phys. Rev. A 89, 032326 (2014).

[17] X.M. Puspus, K.H. Villegas, F.N.C. Paraan, Phys. Rev. B 90, 155123 (2014).

[18] A.R. Plastino, D. Manzano, and J. Dehesa, Europhys. Lett. 86, 20005 (2009).
[19] F. Iemini, R.O. Vianna, Phys. Rev. A 87, 022327 (2013); F. Iemini, T.O. Maciel, R. O. Vianna, Phys. Rev. B 92, 075423 (2015); T. Debarba, R.O. Vianna, F. Iemini, Phys. Rev. A 95, 022325 (2017).

[20] M. Oszmaniec, J. Gutt, M. Kuś, Phys. Rev. A 90, 020302(R) (2014).

[21] M. Oszmaniec, M. Kuś, Phys. Rev. A 90, 010302(R) (2014); Phys. Rev. A 88, 052328 (2013).

[22] G. Sárosi, P. Lévay, J. Phys. A 47, 115304 (2014); Phys. Rev. A 90, 052303 (2014); Phys. Rev. A 89, 042310 (2014).

[23] F. Iemini, T. Debarba, R.O. Vianna, Phys. Rev. A 89, 032324 (2014).

[24] N. Gigena, R. Rossignoli, Phys. Rev. A 92042326 (2015).

[25] A.P.Majtey, P.A.Bouvrie, A.Valdés-Hernández, A.R.Plastino, Phys. Rev. A 93, 032335 (2016).

[26] N. Gigena, R. Rossignoli, Phys. Rev. A 94042315 (2016).

[27] N. Friis, New J. Phys. 18, 033014 (2016).

[28] P. Ring, P. Schuck, The Nuclear Many-Body Problem (Springer, Berlin, Germany, 1980).

[29] S. Hill and W.K. Wootters, Phys. Rev. Lett. 78, 5022 (1997); W.K. Wootters, Phys. Rev. Lett. 80, 2245 (1998).

[30] D. Dasenbrook, J. Bowles, J. Bohr Brask, P.P. Hofer, C. Flindt, N. Brunner, New J. Phys. 18, 043036 (2016).

[31] N. Canosa, R. Rossignoli, Phys. Rev. Lett. 88, 170401 (2002); N. Gigena, R. Rossignoli, Phys. Rev. A 90, 042318 (2014).

[32] R.F. Werner, Phys. Rev. A 40, 4277 (1989).

[33] R. Rossignoli, A. Plastino, Phys. Rev. C 32, 1040 (1985).

[34] R. Bhatia, Matrix Analysis, Springer, NY (1997).

[35] R. Rossignoli, N. Canosa, Phys. Rev. A 67042302 (2003).

[36] P. Hausladen, R. Jozsa, B. Schumacher, M. Westmoreland, W.K. Wootters, Phys. Rev. A 541869 (1996). 